\title{
NIÑAS VÍCTIMAS DE TRATA. DESDE LOS DERECHOS HUMANO A LA ÉTICA DEL CUIDADO
}

\author{
Nuria Cordero Ramos ${ }^{*}$
}

\begin{abstract}
“Mi papá era muy pobre y no tenía dinero para mi boda. Una mujer le recomendó que me mandara a la India para casarme con un hombre de allá. Nos dijo que no teníamos que pagar nada, y que la familia del hombre se encargaría de todo. Nos pareció una oportunidad ideal. Cuando llegué a la India, me vendieron a un prostíbulo". Niña víctima de trata de Bangladesh (OIM)
\end{abstract}

Sumário: 1. Las niñas son víctimas de la trata. 2. Derechos Humanos de las niñas víctimas ¿desde dónde? 3. Aportaciones de la ética del cuidado. Conclusiones.

Resumen: En este artículo exponemos la necesidad de trabajar en la defensa de los derechos de las niñas y adolescentes víctimas de trata y, al mismo tiempo, de diseñar propuestas de intervención basadas en el cuidado en relación con las gravísimas situaciones humanas que estas padecen a causa y como secuelas de dicha execrable vejación. Partimos de la necesidad de evidenciar que la trata tiene diversidad de rostros; uno de ellos, el de ser niña, por su múltiple condición de vulnerabilidad en razón de edad, género, sexo, clase, étnica...Apesar de que no existe acuerdo con las cifras, se sabe que las niñas junto con las adolescentes y las mujeres adultas son las que más sufren esta forma de indignidad. Consideramos que la trata es, sin duda, una expresión de las condiciones de indignidad que supone el quebrantamiento de las nociones más básicas de humanidad. Por ello reivindicamos la necesidad de abordar el tema desde un enfoque crítico y holístico de los derechos humanos, considerados estos no de forma estática, como en realidad lo hace el modelo normativista hegemónico, sino como mediaciones de carácter dinámico al servicio de la dignidad humana. Hacemos explícita la importancia de integrar las aportaciones de la ética de la justicia y la ética del cuidado en el trabajo profesional con estas víctimas. Dicha propuesta está orientada a propiciar actitudes de proximidad, apoyo y empatía, a fin de que las niñas se atrevan a denunciar y narrar las situaciones vividas y de esta forma puedan iniciar rutas de recuperación frente a las profundas huellas que deja la violencia sufrida. La atención a las menores víctimas es una tarea compleja debido al silencio, escasez de denuncias y desconocimiento de las situaciones. Sin embargo, entendemos que la acogida de profesionales orientados desde la ética del cuidado, puede contribuir a evitar sufrimientos silenciados y superar sus daños. Finalmente, en las conclusiones sintetizamos los pasos que pueden concretar la atención a las niñas y adolescentes víctimas de trata. Lo hacemos desde una perspectiva crítica de los derechos humanos y tomando como referencia los aportes de la ética del cuidado. El objetivo final de este trabajo es proponer estrategias humanizadoras que presten atención a la vulnerabilidad que padecen estas niñas, proyectando la dimensión estructural del problema.

Palabras claves: víctimas de trata, niñas, dignidad, derechos humanos y ética del cuidado.

\begin{abstract}
We present the need to work in defending the rights of girls and adolescents trafficked and design proposals based intervention on care attention because of the extreme suffering.We start from the need to demonstrate that human trafficking has a variety of faces, one of them, as a girl, due to their constant vulnerability due to age, gender, sex, class, ethnicity... Although there is no agreement with the figures, it is known that girls, adolescent girls and adult women are the first to suffer this form of indignity. We believe it is undoubtedly an expression of indignity conditions involving the violation of the most basic notions of humanity. Therefore we advocate the need to address the issue of human rights from a complex and inclusive perspective, which are understood as mediations in the service of human dignity.We make explicit the importance of integrating the study of ethics of justice and ethics of care in working with victims. This proposal aims to foster attitudes of closeness, support and empathy for the girls dare to speak and say the situations experienced as a first step to initiate recovery paths. The attention to child victims is a complex task due to the silence and ignorance. However, we understand that the ethics of care provides professional tools to assist in the care and recovery.Finally, the conclusions summarize the steps that can realize the attention to girls who are victims of trafficking. We do it from a critical perspective of human rights and with reference to the contributions of the ethics of care.The ultimate goal of this work is to propose strategies for the humanization of attention to the vulnerability experienced by these girls, projecting the structural dimension of the problem
\end{abstract}

Key words: trafficked, girls, dignity, human rights and ethics of care.

\section{1.- LAS NIÑAS SON VÍCTIMAS DE LA TRATA}

La trata de personas es un fenómeno tan antiguo como la humanidad, pero tan versátil que tiene la capacidad de adaptarse a los nuevos contextos, expuesta siempre al acecho de realidades emergentes muy diversas que le suministren renovadas y constantes víctimas.

\footnotetext{
Universidad Pablo de Olavide (Sevilla). Departamento de trabajo social y servicios sociales. Endereço eletrônico: ncorram@upo.es
} 
El termino trata es la expresión moderna de una toma de conciencia, pero en tanto que problema, según hemos apuntado, tiene carácter histórico:

[...] como problema social comenzó a reconocerse a fines del siglo XIX e inicios del $\mathrm{XX}$ a través de lo que se denominó Trata de Blancas, concepto que se utilizaba para hacer referencia a la movilidad y comercio de mujeres blancas, europeas y americanas, para servir como prostitutas o concubinas generalmente en países árabes, africanos o asiáticos (OIM, 2006: 8).

A partir de este momento, surgieron las primeras hipótesis, considerando que dichos movimientos eran producto de secuestros, engaños y coacciones sobre mujeres inocentes y vulnerables con el objeto de explotarlas sexualmente. Es este un punto de vista marcadamente etnocéntrico: solo toma en consideración como víctimas a las mujeres blancas, olvidando que, con anterioridad a las blanca, ya fueron amplia y largamente compradas, explotadas y sometidas a esa degradación, por obra de comerciantes y conquistadores sin escrúpulos y durante siglos, esclavas negras, indias, etc.

Ateniéndonos como referente conceptual al Protocolo de Palermo ${ }^{1}$, organizaciones como Save the Children, dedicadas a la protección de los derechos de la infancia, explican que "un niño o una niña víctima de trata es cualquier persona menor de dieciocho años que es captada, trasladada, escondida o recibida con fines de explotación desde dentro o fuera de un país, exista o no coacción, engaño, abuso de autoridad o cualquier otro tipo de abuso" (Save The Children y AECID). De esta definición destacamos el elemento etario, es decir, personas menores de edad que se encuentra en una situación de asimetría como respecto a las personas adultas y cuya finalidad es explotarlas valiéndose de su estado de total indefensión.

Aunque no existe acuerdo sobre las cifras, la Organización Internacional para las Migraciones (OIM) estima que, a nivel mundial, cada año aproximadamente un millón de hombres, mujeres, niños y niñas son engañados, vendidos, coaccionados o sometidos a condiciones semejantes a la esclavitud, bajo distintas formas y en diversos sectores: construcción, minería, agricultura y pesca, fabricas de bajo coste de producción, servicio doméstico, prostitución, pornografía, turismo sexual, matrimonios serviles, niños soldados, tráfico de órganos, venta de niños, entre otros. Es de señalar en este punto que las mujeres y, sobre todo, las niñas, constituyen el sector más vulnerable.

Por otra parte, organizaciones como UNICEF, resaltan que según la edad que tengan las niñas, la finalidad de la explotación varía en función de las demandas. Por ejemplo, se dice que las adolescentes son demandadas para la explotación sexual, laboral o con vistas a matrimonios forzados. Sin embargo, las menores con una edad inferior es frecuente que sean elegidas para la mendicidad o en el caso de bebes para las adopciones ilegales. Aunque las niñas de más edad saben que serán utilizadas para prestar servicios sexuales a adultos, no por eso suelen ser conscientes del nivel de abuso y degradación al que se verán sometidas ni del consiguiente daño físico y psicológico que sufrirán.

La vulnerabilidad por razones de edad es reconocida internacionalmente en la Convención sobre los Derechos del Niño (1989), concretamente en los artículos que van del 34 al 40. En ellos se hace constar que los Estados firmantes se comprometen a poner los medios necesarios para impedir el secuestro, venta o trata y proporcionar servicios de apoyo y protección a los niños y niñas que sean víctimas de tortura y privación de libertad. No obstante, conviene resaltar que todos los derechos reconocidos en la Convención Internacional de los derechos del niño y adolescentes son derechos que para ser cumplidos requieren la puesta en práctica de acciones políticas que los reconozcan, haciéndose eco de las responsabilidades que los adultos contraen con los ellos. Para Baratta, dicha convención y su ratificación por un país, antes de producirse constituía un proyecto de país, un proyecto de 
ley. Pero, una vez constituido, dicha convención se convierte, en un proyecto de regulación pública, en un proyecto de sociedad (Baratta,1998: 51).

Las razones fundamentales que justifican esta forma de violencia contra las menores hay que buscarlas en las estructuras de dominación del sistema patriarcal y adulto, las cuales justifican y favorecen la subordinación de las mujeres, y especialmente de las niñas, a los hombres adultos.

La vulnerabilidad por cuestiones de género y sexo se evidencia en múltiples situaciones; pero es de resaltar la existencia de una "demanda femenina" para comercializar en sectores que son en su mayor parte informales, poco protegidos y no regulados, lo que hace que las mujeres, y en particular las menores de edad, se hallen más dependientes de redes de intermediarios, ya sean de tratantes o traficantes (habitualmente, organizados en mafias y redes internacionales). Una de las razones estriba, como señala Villota, en la peculiar y mayoritaria forma de incorporación de las mujeres a la economía a través del trabajo invisible (economía de cuidado) o no remunerado. A ello hay que sumar las desventajas que conllevan circunstancias como las vividas en las últimas décadas, que generan flujos en diferente sentido y un movimiento migratorio desde los países en desarrollo a los industrializados y que cada vez alcanza mayor importancia cuantitativa. (2004: 138).

Es obvio decir que las niñas son víctimas de trata. Y así queda recogido en el "Prefacio" del manual Contra la trata de niños, niñas y adolescentes, elaborado por UNICEF -(2005), que señala: "Cada día, niños, niñas y adolescentes son comprados, vendidos y transportados lejos de sus hogares. La trata de seres humanos es un negocio de miles de millones de dólares que parece estar creciendo. La trata de niños, niñas y adolescentes es ilegal. También es una actividad dañina, ya que los niños, niñas y adolescentes objeto de trata son explotados y sometidos a abusos física y sexualmente". Es difícil obtener datos exactos sobre la trata de niños, niñas y adolescentes, dado su carácter criminal, y porque para los legisladores y los servidores públicos a menudo es difícil reconocer y afrontar la dimensión del problema.

Es preciso advertir que la trata, además de ser un flagrante delito, es un negocio altamente lucrativo que está en crecimiento, debido, de un lado, a la invisibilización del mismo y el desentendimiento de una gran parte sociedad y, de otro, a las dificultades que encuentran las mismas autoridades para reconocer y afrontar el fenómeno y su escasa voluntad en ese empeño.

La convergencia de los factores estructurales mencionados junto con las crisis económicas, los fenómenos migratorios, la pobreza y la exclusión, las guerras, etc., propician que las niñas y adolescentes sean más susceptibles de convertirse en presa de la trata y la explotación en todos los ámbitos del planeta. Estas menores dejan de ser consideradas personas para transformarse en objetos, en mercancías que las cosifica, adquiriendo la condición de puros medios al servicio del lucro y la degeneración de tratantes, intermediarios, en orden a aquellos inhumanos compradores finales interesados en hacerse con ellas y explotarlas.

\section{2.- DERECHOS HUMANOS DE LAS NIÑAS VÍCTIMAS.¿DESDE DÓNDE?}

A partir del año 2000 se inician los esfuerzos a nivel mundial -encabezados por las Naciones Unidas (ONU)- para enfrentar los diferentes tipos de esta forma de explotación humana $^{2}$. Entre los objetivos está el establecer una coordinación efectiva entre países con el propósito de obtener datos veraces y desarrollar y aplicar medidas legales para combatirla. Sin embargo, se constata una impunidad que hace que la oferta y la demanda de personas esclavas se mantengan (MZC 2009: 15), ya que no todas las naciones cumplen y acatan los 
tratados firmados y las leyes nacionales dictadas para combatir la trata no suelen desarrollarse de forma completa o las pautas de las sentencias no se aplican con total rotundidad.

Por esta razón, en la última década se aprecia en numerosos países una preocupación y voluntad crecientes, por parte de las entidades gubernamentales y no gubernamentales, dirigidas a consolidar acciones para combatir, prevenir y mejorar la atención de las víctimas y proteger sus derechos.

Sin embargo, cuando hablamos de derechos humanos se hace imprescindible preguntarse a qué nos estamos refiriendo, pues existe una especie de saturación malsana en la utilización de este término. Así lo explicita Laporta, cuando dice que "empieza a detectarse en la literatura especializada una cierta alarma ante la creciente y la no infrecuente ligereza de apelaciones a los derechos humanos". (Laporta 1989:23). A su vez, Correa Borges (2012) matiza que estos, de hecho, se manejan y proclaman de manera estática y como normas objetivadas, cuando en realidad lo que reclaman, por su misma naturaleza y según su propio espíritu, es un uso crítico, es decir, atento a los cambios históricos y las nuevas formas que adopta el crimen. Por tanto, se espera que los derechos humanos sirvan a sus fines de manera dinámica y operativa, demostrando capacidad para aplicar respuestas adecuadas a todas las circunstancias en las que se detecten vulneraciones de la dignidad humana. El propio Bobbio (1981:10) señalaba que la preocupación por los derechos humanos no debería centrarse en su justificación sino en su protección.

Compartimos la misma preocupación en el tema que nos ocupa, puesto que las referencias a los derechos humanos son continuas desde diferentes instancias, pero olvidando, o sin hacer explícitos, los enfoques y abordajes desde donde se deben concebir y cumplir.

Este constatación se deja ver claramente en Costa Rica ${ }^{3}$. A partir de la investigación que hemos llevado a cabo para conocer la realidad de la trata, tomando nota del trabajo desarrollado por las instituciones gubernamentales y no gubernamentales, verificamos que en la defensa de los derechos humanos de las víctimas dominan los discursos institucionales. Dichos discursos hacen referencia, fundamentalmente, al conocimiento y cumplimiento de las normativas internacionales, a la importancia de la capacitación en materia legal por parte de los profesionales y a la necesidad de trabajar en colaboración con las fuerzas de seguridad del Estado.

Este enfoque grandilocuente y burocrático adolece a su vez de grades incongruencias ${ }^{4}$, entre ellas la relativa a las escasas garantías que se ofrecen en orden a la protección de los derechos reconocidos por la normativa internacional y nacional. En el caso de las víctimas menores, en Costa Rica no existen recursos ${ }^{5}$ públicos institucionales que puedan protegerlas con plenas garantías.

Las principales limitaciones del enfoque normativo en el problema de la trata de menores es que pone especial énfasis en los aspectos punitivos y criminológicos, donde se prima la persecución del delito y la condena de los tratantes. En este sentido, y en cuanto a las medidas de protección a las víctimas, fundamentalmente se centra en la importancia de que denuncien, pero sin tener en cuenta la incidencia de los factores estructurales en cada caso concreto y desde un desconocimiento de las realidades étnicas, culturales, familiares... de las niñas.

Tal forma de ver las cosas, reguladora y punitiva, hace alianza con el fenómeno migratorio, donde la trata y el tráfico de personas parecen confundirse. La distinción es importante puesto que el "tráfico de personas" es un delito convencional, aunque de ejecución compleja y organizada, que incumple la legislación interna de los países, particularmente en materia migratoria, mientras que la "trata de personas", es un crimen de lesa humanidad que violenta flagrantemente los derechos humanos, socavando la integridad y dignidad de las personas y los pueblos que la padecen. 
Dicha confusión, paradójicamente, otorga el protagonismo a las fuerzas de seguridad y propicia que los operadores jurídicos adopten un papel mediante el que las víctimas menores de edad corren el riesgo de ser invisibilizadas o, lo que es peor, de ser cosificadas, de convertirse de nuevo en meros objetos, pero esta vez al servicio no ya de los violadores sino del propio sistema judicial. De esta forma, se olvida el hecho principal, esto es, que por encima de todo está en juego la protección de personas inocentes, vulnerables y gravemente vulneradas. Coincidimos con García (2009) en señalar que estos dos enfoques, el migratorio y el normativo, son los hegemónicos en el abordaje de la trata, siendo necesario incluir, según la propia autora, la perspectiva dinámica de los derechos humanos.

Por supuesto, somos conscientes de la necesidad de conocer las normativa por parte de los profesionales que trabajan en las instituciones dedicadas a la atención de las víctimas, así como la importancia de la colaboración de la sociedad civil con cuerpos y fuerzas de seguridad para generar acciones de lucha contra los tratantes y victimarios. Pero, asimismo, estamos convencidos de que el abordaje desde los derechos humanos debe hacerse mediante "una concepción mucho más compleja, relacional, socio-histórica y holística que priorice las propias prácticas humanas que son las que realmente hacen y deshacen, construyen y deconstruyen derechos humanos y sobre las cuales se inspiran y elaboran teorías" (Sánchez 2009: 2).

Desde esta perspectiva crítica, los derechos humanos son, como defendía el fallecido Joaquín Herrera (2005), "espacios de lucha por la dignidad humana". Entendemos ${ }^{6}$ que son procesos sociales, económicos, normativos, políticos y culturales que abren, favorecen y consolidan, desde el reconocimiento, la transferencia de poder y la mediación jurídica, las particulares concepciones de la dignidad humana. Lo dicho refuerza la reiterada advertencia de que las leyes y las normas son consideradas instrumentos de exigibilidad y de garantía, pero no aseguran por sí mismas la obligatoriedad y cumplimiento del derecho, a no ser que haya una presión política y social sobre el carácter vinculante de estas normas.

Esta forma de entender los derechos humanos permite transitar por un camino que va de lo concreto a lo abstracto y de lo particular a lo general ${ }^{7}$. Un camino cuyos itinerarios convergen de tal forma que el valor general de la dignidad presente en la Declaración Universal de los Derechos Humanos alcanza su contenido cuando se concreta de forma efectiva mediante las condiciones de posibilidad de la propia dignidad que pueden ofrecer a cada una de las niñas víctimas de trata.

Desde el enfoque de los derechos humanos que venimos defendiendo, las niñas víctimas de trata forman un colectivo porque comparten perfiles y elementos comunes como el engaño, la coacción, la explotación..., Pero eso no quiere decir que sean consideradas como una categoría standard, sino que deben ser atendidas una por una, es decir, como sujetos con nombre propio, identidad, procedencia y situaciones jurídicas, sociales, familiares y culturales particulares que han ser cuidadosamente explicitadas.

\section{3.- APORTACIONES DE LA ÉTICA DEL CUIDADO}

Multitud de estudios corroboran que las menores, víctimas de otras formas de violencia (doméstica, acoso escolar, ciberbulling...), suelen silenciar las situaciones que padecen, condenándolas al desamparo y dejando en ellas graves secuelas físicas y psíquicas. Si esto es así, cuánto más sucederá en el caso de aquellas que son víctimas de trata.

Tal como venimos diciendo, la defensa de los derechos de las niñas y adolescentes víctimas de la trata no debe limitarse a la aplicación de garantías legales o a la activación de recursos asistenciales, que sin duda son necesarios, sino que, por su múltiple condición de vulnerabilidad, es necesario fundamentar las acciones dirigidas a ellas sobre un marco axiológico que permita regenerar las capacidades y el reconocimiento propio y ajeno de su 
dignidad, nunca perdida. Esto quiere decir, utilizando la terminología de los tratadistas, que formando un todo con la ética de la justicia explícita en los derechos humanos, es necesario integrar a la ética del cuidado. Ambas, como vienen sosteniendo a lo largo de su obra Victoria Camps (1990, 1998, 2005), han de complementarse. A su vez Mayorga (2009: 110) insiste de forma convincente en que "el imperativo práctico exige tratar con respeto a los seres libres y con cuidado a los seres vulnerables, sintetizando perfectamente lo mejor de las aportaciones de la ética del cuidado y de la ética de la justicia".

Es evidente, en efecto, que al otorgar rango moral a los sentimientos, como hace la ética del cuidado, se enriquece el pensamiento ético y se establecen bases sólidas para la virtudes públicas. Así pues, las razones que sitúan a la ética del cuidado como referente de importancia para la intervención con menores que han sufrido tanta violencia, las podemos encontrar en la propia etimología del término, puesto que el cuidado puede ser considerado como cura.

En su forma más antigua, cura en latín se escribía coera y era usada en un contexto de relación de amor y de amistad. Expresaba actitud de cuidado, de desvelo, de preocupación y de inquietud por la persona amada o por el objeto de estimación. También en la actualidad adquiere un sentido más terapéutico que hace referencia al interés por la suerte del congénere.

La ética del cuidado suele identificarse con algunas perspectivas feministas seguidoras de Gilligan (1985), que defienden las diferencias morales entre los hombres y las mujeres a partir de los valores de la justicia y del cuidado. Para esta autora, las mujeres construyen una moralidad vinculada con el cuidado porque su desarrollo moral pasa por la comprensión de la responsabilidad y por las relaciones mutuas, de ahí que, para ellas, el imperativo moral de las mujeres sea el cuidado, mientras que el de los hombres es más un mandamiento para respetar los derechos y la justicia.

$\mathrm{Si}$ atendemos a este planteamiento, parece que está refiriéndose a dos tipos de comportamientos éticos: el propio de la "ética de la justicia", específico de los varones, y el de la "ética del cuidado", más adecuado a las mujeres (Gilligan, 1985: 266). Sin duda, la distinción en el campo de la ética ha cuestionado y enriquecido muchos de sus principios éticos existentes durante siglos, y tiene una gran incidencia en las actuales vertientes de la Ética Aplicada. Pero, por otra parte, consideramos las identidades de género que estadísticamente caracterizan a los humanos de uno u otro sexo como resultado del sistema estratificado de género en que nos socializamos, el cual tiene un peso determinante en su modelación con fines de dominio de unos por otros. Por tanto, si desde el punto de vista de la ética las virtudes el cuidado tienen un valor positivo y primordial, será necesario universalizarlo, convirtiéndolo en una obligación de todos: hombres y mujeres.

La importancia de cumplir con esa obligación, actuando desde la ética del cuidado exige, tal y como apunta Mayeroff (1971), ayudar a otra persona a crecer. Genera un proceso que implica, siguiendo a este autor, dedicación, confianza, paciencia, humildad, honestidad, conocimiento del otro (alteridad) y coraje. También, pues, en la visión de Mayeroff, los valores morales son inherentes al proceso de cuidar y crecimiento.

Este proceso de construcción de la ética del cuidado reclama, siguiendo a Noddings (1984), que la persona que lo practica se hace cargo del bienestar, la protección o el mantenimiento de algo o de alguien. Es equiparable, según esta autora, a tener un impulso o deseo de inclinación hacia otros. Asimismo, la importancia del cuidado para esta autora radica en que la realidad del otro se presenta como una posibilidad para sí mismo, es decir, que cuidar de otros es cuidar de uno mismo.

Por todo lo dicho, hay que sortear la falsa apreciación de que el cuidado es sólo una práctica que ha de ejercerse en el ámbito doméstico, sino que es necesario que se ejerza en el ámbito público, prioritariamente en las instituciones destinadas a prestar ayuda a las personas vulnerables de la sociedad. Aportamos en este punto las reflexiones de Rubio Carracedo: "La 
ética es constitutivamente social y, por consiguiente, pública. Y, aunque no sea tan sencillo definir claramente el espacio público, nos basta su caracterización negativa por J.S. Mill como el espacio donde puede causarse daño a los demás (Harme Principle) para comprender que es allí donde se sitúa la ética" (1992: 234-235).

Desde la ética del cuidado se pretende humanizar las relaciones institucionales y conseguir que el valor de la dignidad humana, tal y como se reconoce en la Declaración Universal de los Derechos Humanos y en las constituciones de la mayor parte de los países firmantes, se pueda concretar en gestos y acciones profesionales.

La propuesta de actuar de la ética del cuidado con las menores que han sido violentadas por la trata, incide en el cómo. Quiere alejarse de estrategias profesionales diseñadas desde un punto de vista exclusivamente terapéutico o legal, de forma abstracta y descontextualizadas, centradas en la aplicación de protocolos que tratan de calzar las realidades en formalismos burocráticos y cuantificables, dejando de lado el encuentro personal. Se trata más bien de crear y recrear propuestas de acción, inductivas, contextualizadas y personalizadas, que posibiliten el acompañamiento en las situaciones trágicas y dramáticas que están viviendo.

La construcción de una relación de empatía, establecimiento de alianzas dialógicas, la búsqueda de un contexto acogedor que permita la relajación y la comodidad, la actitud de escucha, el respeto de los silencios, la señalización de los indicadores de resiliencia y la visibilización de los factores de autoprotección y empoderamiento, son algunos de los elementos relevantes a tener en cuenta en las intervenciones con las personas víctimas de la trata, y de manera especial con las menores de edad. Estos son signos visibles del accionar desde la ética del cuidado que están presentes en algunas de las instituciones que trabajan en la atención de niñas víctimas de trata.

El nivel de detección y identificación de una niña como víctima es sumamente importante para poder activar la asistencia y protección necesaria. Este trabajo inicial es sin duda la base para poder llevar a cabo todo el proceso de recuperación y, si fuera el caso, de denuncia. Consideramos fundamental la capacitación profesional en técnicas que faciliten la detección y la atención de las niñas, desde las premisas del cuidado en ámbitos como servicios sociales, salud, educación, justicia y cuerpos y fuerzas de seguridad entre otros

En buena parte de las ocasiones ni siquiera son conscientes de las situaciones de indignidad que están sufriendo o, si lo son, temen las represalias de las redes de trata, contra ellas y sus familias. Pero este silencio también tiene que ver con los procedimientos institucionales a los que se ven sometidas las menores, en caso de que se decidan a revelar la situación. Desde la ética del cuidado se propone humanizar los procedimientos, para que resulten más amigables a las niñas y adolescentes, evitando actitudes que las revictimicen y estigmaticen aún más.

La relación entre profesional y la menor de edad, debe abordarse desde el marco axiológico del cuidado, en un cuerpo a cuerpo que muestre respeto ${ }^{8}$, con la finalidad de que las menores puedan sentir confianza y puedan comenzar a verbalizar el daño recibido. La acogida del sufrimiento es punto de partida de la relación profesional y se presenta como posibilidad para que las niñas puedan percibir que son escuchadas y comprendidas.

El cuidado como valor que guía la atención con las víctimas de trata permite que afloren los sentimientos de impotencia, a fin de liberarlas de toda culpabilidad y responsabilidad en el padecimiento. La finalidad es conseguir que desarrollen todas sus potencialidades como personas, siendo necesario trabajar al mismo tiempo para que la sociedad las incluya y las reconozca como personas válidas y competentes. 


\section{CONCLUSIONES}

Para finalizar, y modo de conclusión, enumeramos de forma sintética acciones que desde el enfoque crítico de los derechos humanos y de la ética del cuidado se pueden llevar a cabo en la adecuada atención a las niñas víctimas de trata:

1.- VISIBILIZAR: Como primer paso es necesario hacer visible el problema en el sentido más literal, debido al desconocimiento que existe en general ante la complejidad del fenómeno de la trata y las razones que hacen que se cebe de forma especial en niñas y adolescentes. Su importancia también está en reconocer las razones estructurales que las convierten en víctimas invisibles.

2.- PREVENIR: Radica en proponer medidas preventivas que comiencen por una deconstrucción de mitos y prejuicios sociales que dan pábulo a tolerar conductas consistentes en que las niñas en concreto, al igual que las demás personas sea cual fuere su condición, puedan ser explotadas y tratadas como mercancías. Además, es necesario activar en el interior de los contextos sociales propicios a generar vulnerabilidad y negativas condiciones de vida en relación con niñas y adolescentes, todo tipo de precauciones, trabajando en la sensibilización de las personas y buscando alternativas que ayuden a mejorar el medio en el que viven.

3.- GARANTIZAR: Activar políticas públicas que les permitan vivir en entornos seguros y protectores. Las niñas víctimas de trata sufren graves alteraciones, entre otras, la separación de su familia, el abandono del sistema educativo, daños físicos, emocionales y psicológicos, rechazo, etc. . La aplicación de las medidas legales deberá garantizar el cuidado de las menores, proponiendo la activación de recursos materiales para alimentación, alojamiento, educación y salud, junto con cuidados y tratamientos psicológicos. De3 forma tal que las acciones de ayuda y cuidado activadas mediante recursos institucionales, estén encaminadas a minimizar la ansiedad, el miedo y las situaciones de vulneración de derechos que afectan seriamente a sus capacidades para poder desarrollarse como personas.

4.- DIGNIFICAR: Se trata de trabajar de forma personalizada par reparar e intentar recuperar la dignidad perdida. Hay que ejercer el cuidado en la tarea de aliviar el sufrimiento y compensar las pérdidas sociales, morales y materiales, evitando sobre todo la revictimación.

La reconstrucción de la historia de daños vividos y silenciados será más viables si se facilita el encuentro dialógico entre profesionales y víctimas. La atención será considerada como un proceso para la reparación de daños materiales y simbólicos, encaminados a desarrollar sus capacidades en el futuro, de modo que se generen condiciones para el desarrollo de un vida digna.

5.- EMPODERAR: Consiste en ponerlas en valor, tomando el sentido que otorga Lagarde (2004) al empoderamiento de las mujeres. En el caso que nos ocupa, el empoderamiento, además de tener un sentido preventivo para evitar que niñas y adolescentes sean objeto de trata, posee un gran potencial, sobre todo para aquellas que consigan narrar los daños recibidos, denunciar e iniciar itinerarios de recuperación.

\section{REFERENCIAS BIBLIOGRÁFICAS}

BOBBIO, N. El tiempo de los derechos .Madrid: Sistema, 1991.

BENHABIB, S. EI Ser y el Otro en la ética contemporánea. Barcelona: Gedisa, 2006. 
BARATTA, A. Infancia y Democracia. En: García Mendez, Emilio y Belfo, Mary Comp Infancia, Ley y Democracia en América Latina. Buenos Aires: Editorial Temis, Depalma, 1998.

CAMPS, V. Virtudes públicas. Madrid, Espasa, 1990.

El siglo de las mujeres. Madrid, Cátedra, 1998.

La voluntad de vivir. Barcelona, Ariel, 2005.

CEPAL. Derechos humanos y trata de personas en las Américas. Resumen y aspectos destacados de la Conferencia Hemisférica sobre Migración Internacional. Serie Seminarios y Conferencias N. 33. Santiago de Chile, 2003.

CORDERO, N., CRUZ, P. y SOLORZANO, N. (2012) Trata de personas, dignidad y derechos humanos. Sevilla: Arcibel Editores.

CORREA BORGES, P.C. A Tutela Penal dos Direitos Humanos. Revista Espaço Académico, n. 134, 2012.

GARCIA, S. "Violencia de género y tráfico de mujeres: la explotación social de las traficadas" en Documentación Social ${ }^{\circ} 153$ Sociedad civil y nuevos movimientos sociales. Madrid: Cáritas española, 2009.

GILLIGAN, C. La moral y la teoría. Psicología del desarrollo femenino. México: Fondo de Cultura Económica, 1985.

GOFFMAN, I. Estigma. La identidad deteriorada. Buenos Aires: Amorrortu, 1986.

LAGARDE. M. Mujeres cuidadoras: entre la obligación y la satisfacción, Congreso Internacional SARE 2003: "Cuidar Cuesta: costes y beneficios del cuidado", VictoriaGasteiz: EMAKUNDE-INSTITUTO VASCO DE LA MUJER, págs. 155-160, 2004.

HERRERA, J. Los derechos humanos como productos culturales. Madrid: Catarata, 2005.

MAYEROFF, M. On Caring. New Cork: Harper and Row, 1971.

MARORGA, F. La fórmula del bien. Madrid, Éride Ediciones, 2009.

NODDINGS, N. Caring: A feminine approach to morality and moral education. Berkeley: University of California Press, 1984.

NACIONES UNIDAS. Protocolo de Palermo. Protocolo para Prevenir, Reprimir y Sancionar la Trata de Personas, especialmente de mujeres y niños, que complementa la Convención de las Naciones Unidas contra la delincuencia organizada transnacional. Palermo: Naciones Unidas, 2000.

OIM. La trata de personas: Aspectos básicos. México: Comisión Interamericana de Mujeres de la Organización de Estados Americanos, Organización Internacional para las Migraciones, Instituto Nacional de Migración, Instituto Nacional de las Mujeres, 2006. 
RUBIO CARRACEO, J. Ética constructiva y autonomía personal. Madrid: Tecnos, 1992.

SANCHEZ, D. Contra una cultura estática de los derechos humanos, 2009. Disponible en: http://www.pensamientocritico.info/articulos/otros-autores/213-contra-una-culturaestatica-de-derechos-humanos.html.

\author{
SAVE THE CHILDREN y AGENCIA ESPAÑOLA DE COORPERACIÓN \\ INTERNACIONAL PARA EL DESARROLLO (AECID). El delito de trata. Disponible en: \\ http://violenciaytrata.savethechildren.es/trata.php.
}

\title{
SENNETT, R. El respeto. Sobre la dignidad del hombre en un mundo de desigualdad. Barcelona: Anagrama, 2003.
}

UNICEF- Unión interparlamentaria. Contra la trata de niños, niñas y adolescentes. Serie manual para parlamentarios, $\mathrm{n}^{\circ}$ 9, 2005.

VILLOTA, P. Globalización y Desigualdad de Género. Madrid: Editorial Síntesis, 2004.

\footnotetext{
1 "Protocolo para Prevenir, Reprimir y Sancionar la Trata de Personas", especialmente de mujeres y niños, que complementa la Convención de las Naciones Unidad contra la delincuencia organizada transnacional, Naciones Unidad, 2000.

${ }^{2}$ Ese año se celebró la Convención contra la Delincuencia Organizada Transnacional y se elaboraron tres protocolos, precisamente uno de ellos, el conocido como el Protocolo de Palermo, está destinado a prevenir, reprimir y sancionar la trata de personas, especialmente mujeres y niños (ONU, 2000: 2).

${ }^{3}$ En el año 2011 realizamos una investigación sobre trata de personas en Costa Rica un equipo formado por miembros de la UPO (España) y del IDESPO (Costa Rica). Para conocer los detalles se puede consultar la obra Trata de personas, dignidad y derechos humanos, mencionado en las regencias bibliográficas.

${ }^{4}$ Susana Chiarotti afirma que la "mayoría de los países no han ratificado el Protocolo para Prevenir, Reprimir y Sancionar la Trata de Personas, Especialmente Mujeres y Niños, pero incluso los que lo han firmado todavía no han provisto los mecanismos nacionales y las leyes adecuadas para investigar, procesar y sancionar estos delitos, proteger a las y los testigos y buscar reparaciones para las víctimas." (En CEPAL, 2003: 54)

${ }^{5}$ Esta afirmación la hacemos a partir de la información obtenida de la entrevista realizada en Agosto 2011 a responsables del PANI

${ }^{6}$ Desde dónde nos situamos para comprender los derechos humanos, se desarrollada más ampliamente en la obra del autor citado, Trata de personas, dignidad y derechos humanos.

${ }^{7}$ La expresión que utiliza Benhabib (2006) para ir de lo general a lo particular es el otro abstracto y el otro concreto. Lo recoge de la siguiente manera:

"Veo la relación del otro generalizado con el otro concreto de acuerdo con el modelo de un continuo. En primer lugar está el compromiso universalista con la consideración que merece todo individuo humano como merecedor de respeto de la moral universal [...] El punto de vista del otro concreto, está implícito en aquellas relaciones éticas en las que siempre nos hallamos inmersos en el mundo de la vida real. Ser miembro de una familia, padre, cónyuge, hermana o hermano, significa saber cómo razonar desde el punto de vista del otro concreto" (Benhabib, 2006: 23).

${ }^{8}$ Tomamos como referente las palabras de Sennett (2003) sobre el respeto: "La conducta que expresa respeto es a menudo escasa y está desigualmente distribuida en la sociedad, pero el significado del respeto es social y psicológicamente complejo. Como resultado, los actos que vehiculan el respeto -los actos de reconocimiento de los otros - son exigentes y oscuros" (Sennett, 2003: 70).
} 